\title{
Treatment of post-orthodontic white spot lesions with CPP-ACP paste: A three year follow up study
}

\author{
Said KARABEKIROĞLU ${ }^{1}$, Nimet ÜNLÜ1 ${ }^{1}$, Ebru KÜÇÜKYILMAZ², Sevgi ŞENER ${ }^{3}$, Murat Selim BOTSALI ${ }^{4}$ \\ and Sıddık MALKOÇ5
}

\author{
${ }^{1}$ Department of Restorative Dentistry, Necmettin Erbakan University, Konya, Turkey \\ ${ }^{2}$ Department of Pediatric Dentistry, Izmir Katip Celebi University, Izmir, Turkey \\ ${ }^{3}$ Department of Oral Radiology, Necmettin Erbakan University, Konya, Turkey \\ ${ }^{4}$ Department of Pediatric Dentistry, Selçuk University, Konya, Turkey \\ ${ }^{5}$ Department of Orthodontics, Inönü University, Malatya, Turkey \\ Corresponding author, Said KARABEKIROĞLU; E-mail: dentisaid@hotmail.com
}

\begin{abstract}
To determine the efficacy of topical applications of $10 \%$ casein phosphopeptide-amorphous calcium phosphate (CPP-ACP) paste in reverting white spot lesions (WSLs) after fixed orthodontic treatment. Forty one participants were randomly assigned to either the test group (CPP-ACP) or the control group (only fluoride toothpaste). All patients used regular fluoride-containing toothpaste. CPPACP paste or fluoridated toothpaste was applied on to tooth surfaces with WSLs twice a day during 36-month after de-bonding. The labial/buccal surfaces of teeth were assessed by use of DIAGNOdent pen (DD), Gorelick Index and ICDAS II criteria at baseline (T1) and 36-month (T2) follow-up visits. The total counts of Streptococcus mutans and Lactobacillus were obtained at T1 and T2. DMFT, DMFS, stimulated flow rate and buffer capacity were calculated in two appointments. Daily usage of CPP-ACP paste was not better than normal care for improving the appearance of WSLs after 36 months.
\end{abstract}

Keywords: Conservative dentistry, Remineralization, CPP-ACP

\section{INTRODUCTION}

White spot lesion (WSL) is a common iatrogenic effect seen in patients undergoing orthodontic treatment with fixed appliances ${ }^{1-4)}$. The presence of fixed orthodontic appliances further affects oral hygiene and makes the cleaning of teeth more difficult; hence, accumulation of plaque around brackets and bands increases, which causes enamel demineralization ${ }^{3,5}$. The naturally occurring self-cleansing mechanisms of the oral musculature and saliva are limited by the irregular surface of brackets, bands, and wires. The composition of the bacterial flora of the plaque shows a rapid shift following the placement of orthodontic appliances ${ }^{3)}$. The general belief that these lesions disappear after removal of the fixed appliances is controversial, and in many cases these lesions remain visible as a permanent enamel scar ${ }^{5}$. An aim of modern dentistry is focused on a prophylactic approach, instead of invasive restoration, of carious defects. Management of WSL should involve methods of both preventing demineralization and encouraging the remineralization of existing lesions. If salivary or plaque calcium, phosphate ions, and fluoride ions are present in adequate amounts, they can also promote the remineralization of previously demineralized enamel ${ }^{4-6}$. Several methods have been used to prevent or reduce enamel demineralization during orthodontic treatment, including fluoride application in various forms, enamel sealants, rigorous oral hygiene regimens, use glass ionomer cement for bonding brackets and modified appliance designs ${ }^{6-10)}$. There have been only a few studies related to the possibility of improvement or even 'cure' of white spots after de-bonding, with or without extra measures ${ }^{4,55}$.

Recently, casein phosphopeptide-amorphous calcium phosphate (CPP-ACP) derived from milk protein casein has been reported to reduce demineralization of the tooth structure and enhance remineralization ${ }^{11}$. CPP is believed to have an antibacterial and buffering effect on plaque, and interfere in the growth and adherence of Streptococcus mutans and Streptococcus sobrinus. Moreover, CPP can stabilize the level of ACP in saliva by preventing precipitation of calcium and phosphate, and stabilize the level of calcium ${ }^{12)}$. Tooth Mousse/MI Paste (GC, Tokyo, Japan) is a topical remineralizing cream containing CPP-ACP (10\% w/v). While in vitro studies of the cream have shown demineralization prevention and remineralization promotion, its efficacy has to be substantiated in different short term clinical trials ${ }^{11-16)}$. Therefore, the aim of this project was to evaluate the efficacy of topical applications of $10 \%$ CPP-ACP paste in reverting white spot lesions (WSLs) in a post-orthodontic population in a 36 -month.

\section{MATERIALS AND METHODS}

\section{Study design}

The study had a single blinded-randomized, controlled design with two parallel groups. Approval for the study was obtained from the regional ethics committee of Selcuk University Dentistry Faculty (2010/08-1). The patients and their parents were informed about the clinical study and signed informed consent forms. The patients were randomized using an internet-based 
computer program. Subjects were evaluated at clinically each 6 month for three years, but outcomes were obtained only baseline and after 36 months. The primary endpoint measure was laser fluorescence (DIAGNOdent pen (DD), KaVo, Biberach, Germany) readings performed at baseline (just after de-bonding) and after 36 months and the secondary endpoint was a Gorelick Index+ICDAS II Criteria as described below ${ }^{17,18)}$. The subject caries experience and salivary variables also evaluated in two appointments.

\section{Participants}

This study was carried out on forty nine adolescents, aged 14-20 years, who exhibited at least four WSLs at de-bonding time. They were chosen from a number of patients who had just finished their orthodontic treatment with full fixed appliances. All patients were treated with 0.018 inch slot MBT fixed orthodontic appliances (Equilibrium ${ }^{\circledR} 2$, Dentaurum, Ispringen, Germany), and their teeth were bonded with a lightcured composite resin and adhesive (Transbond XT, 3M Unitek, Monrovia, CA, USA). The patients with any systemic disease, smoking habits, plaque accumulation and periodontal disease, enamel hypoplasia, dental fluorosis or intrinsic and extrinsic pigmentation, no brushing habits before bedtime, WSL at bonding, restoration or cavity in any anterior teeth, high caries activity (such as low saliva secretion rates, DMFT>10) and who had secondary orthodontic treatment before were excluded from the study $(n=8)$. All subjects lived in a community with no fluoride supply. Sample size calculations were based on detecting a difference of $30 \%$ reduction in DD reading between the test group and the control group using a two-tailed significance level of $5 \%$ with an $80 \%$ power. A total sample size of 180 teeth (90 teeth per group) would give more than $80 \%$ power to detect significant differences with a 0.25 effect size between two groups and at a $p=0.05$ significance level.

\section{Treatment groups}

All patients received the usual home-care oral hygiene instructions and a packet with nonprescription fluoride toothpaste (1,450 ppm of fluoride; Colgate Total, 1,450 ppm F, São Paulo, Brazil), a manual toothbrush, and dental floss. A single blinded study design was applied and there was no allocation concealment. The special plastic tubes were packed, and only the subjects could identify test or placebo packets. The placebo packets were comprised only fluoridated toothpaste $(1,450$ ppm). Participants in the test group received the remineralizing cream (Tooth Mousse, GC Asia, Tokyo, Japan) containing 10\% w/v CPP-ACP, while participants in the placebo control group received only brushing standard fluoride toothpaste (Colgate, 1,450 ppm F). In test group, participants applied the topical cream CPPACP (Tooth Mousse, GC) to the tooth surfaces by using a clean finger twice a day, after brushing their teeth with fluoride toothpaste for a period of 36 month. For areas that are difficult to reach (between the teeth), patients were advised to use an interproximal tooth-cleaning brush. Eating and drinking were prohibited for 30 min after application. Those in the control group were informed and encouraged to use the fluoride toothpaste two times a day (morning and evening) during the study period. At each 6 month visit, participants received a prophylaxis and oral hygiene reinforcement during three years.

\section{Measurements}

1. Caries experience

Caries experience (DMFT and DMFS) were performed for each subject at baseline and after 36 months. Caries experience was evaluated as DMFT (decay, missing and filling teeth) and DMFS (decay, missing and filling teeth surfaces) to assess the patient's risk level, based on clinical and radiographic findings. The WHO criteria were utilized to diagnose the carious status of the subjects ${ }^{19)}$. Clinical examination was made using a plane mouth mirror and blunt, sickle probe with the aid of a dental chair light on dried teeth by one examiner (S.K). The sickle probe was used to remove debris, check restoration margins and detect cavitation. In clinical examination, diagnosis of caries was made only when there was clear evidence of loss of tooth substance.

The digital bitewing images were obtained using the same intraoral unit (Trophy CCX Digital periapical X-ray Machine, France) using number 2 Digora phosphor plates at $65 \mathrm{kV}, 8 \mathrm{~mA}$. After the plates were exposed, plates were processed by Soredex Digora Optime, France. The clinical and radiographic data were recorded separately for each subject by the same examiner. All radiographs included mesial surface of the first premolar and the distal surfaces of the second molar and no artifacts, position or processing errors. All approximal surfaces can be observed clearly in bitewings. All teeth that have carious lesion were restored with resin composite or amalgam at baseline.

\section{Visual inspection}

WSL severity and activity status was recorded at each two point (baseline and 36th month). The Gorelick WSL index ${ }^{17)}$ (score $0=$ no white spot formation, score $1=$ slight white spot formation, score $2=$ severe white spot formation, and score $3=$ white spot formation with cavitation) was used with the aid of standard $3.0 \times$ loupes (Keeler, Windsor, UK) for visual severity evaluation of the buccal surfaces of the anterior teeth, premolars, and first molars in the upper and lower jaws. Only teeth that shown mild or severe lesion were evaluated for this study. Each lesion was also scored according to ICDAS II free smooth-surface criteria for activity situation ${ }^{18)}$ (Active lesion: Surface of enamel is whitish/yellowish opaque with loss of luster; feels rough when the tip of the probe is moved gently across the surface. Inactive Lesion: Surface of enamel is whitish, brownish or black. Enamel may be shiny and feels hard and smooth when the tip of the probe is moved gently across the surface).

Scoring was performed under direct illumination using a dental lamp after light pumicing and drying with compressed air for $5 \mathrm{~s}$. Measurements were performed on 
all patients who were enrolled in the study by the same operator. To assess the reproducibility of the diagnostic criteria application, the intra-examiner calibration was performed. An intra-examiner test was conducted by reexamining 10 randomly selected teeth one hour after the first examination. The level of intra-examiner agreement was measured using Cohen's kappa statistics. Intraexaminer agreement for caries detection was good with a Kappa value of $90 \%$. The progression or regression status of each WSL was based on a labial surface comparison between the time points. The following scores were given: Score 0 (unchanged-stable WSL); Score+(improved-regressed-WSL) and Score-(worsenedprogressed WSL). Each score also was recorded for WSL changing from active to inactive or vice versa.

\section{Laser fluorescence}

Status of the WSLs were assessed using a DD at baseline and 36th month visit, which had been shown to have similar reliability and validity as the conventional DD laser-induced fluorescence detection device when quantifying changes in carious lesion in teeth. DD was calibrated according to the manufacturer's instructions before every use. All teeth were cleaned and dried by air syringe before using the DD under cotton roll isolation. The peak reading displayed on the panel of the DD during the scan was recorded for each tooth surface. The same examiner made all measurements for 3 times to eliminate the operator effect for each lesion and was calibrated before the study in the use of DD in line with the manufacturer's directions.

\section{Salivary variables}

Samples were collected in the morning between 9 and 12 a.m. under standardized conditions. Stimulated saliva was collected while chewing on a piece of paraffin wax for $5 \mathrm{~min}$ in a test tube graduated in milliliters, and the saliva secretion rate was expressed as milliliters per minute $(\mathrm{mL} / \mathrm{min})$. During collection, the patient was in an upright position. The buffer capacity of stimulated saliva was determined using the CRT Buffer ${ }^{\circledR}$ strip (Ivoclar-Vivadent, Schaan, Liechtenstein). After $5 \mathrm{~min}$ of reaction, a comparison was made with the colored chart provided by the manufacturer and the buffer capacity was scored as low ( $\mathrm{pH}$ below 4$)$, medium $(\mathrm{pH}$ between 4.5 and 5.5) or high (pH over 6 ). The fresh saliva sample was then used to determine MS and LB counts. The CRT ${ }^{\circledR}$ Caries Risk Test (Ivoclar-Vivadent) was used to record the salivary counts of MS and LB. Both agar surfaces were wetted with saliva using a pipette without scratching the agar surface. The test vial was placed upright in the incubator (Ivoclar-Vivadent) and incubated at $37^{\circ} \mathrm{C} / 99^{\circ} \mathrm{F}$ for $48 \mathrm{~h}$. After removal of the vial from the incubator, the density of the MS and LB colonies were compared with the corresponding evaluation pictures in the enclosed model chart $(0=0-$ $\left.<10^{3}, 1=10^{3}-10^{4}, 2=10^{4}-10^{5}, 3=>10^{5} \mathrm{CFU} / \mathrm{mL}\right)$.

\section{Statistical analysis}

The statistical analysis was processed with the SPSS
17.0 software system (SPSS, Chicago, IL, USA). A $p$-value of $<0.05$ was considered statistically significant. Descriptive statistics, including the means, standard deviations and frequencies (percentages), were calculated. Since, the results of Kolmogorov-Smirnov and Shapiro-Wilk tests demonstrated that the data were normally distributed $(p>0.05)$, parametric tests were used for statistical analysis. Monte Carlo Exact Chi-Square test was used for evaluation of two groups with Mutans Streptococcus, Lactobacillus and buffer capacity levels. Exact Marginal Homogeneity Test was used same three parameters (Mutans Streptococcus, Lactobacillus and buffer capacity levels) for define differences about two time points. The follow-up DD readings, DMFT, DMFS and stimulated saliva rate were compared with baseline within each group with the aid of two-way repeated measure ANOVA. For all ILs, the progression or regression scores were analyzed by means of a proportional odds ordinal logistic regression model.

\section{RESULTS}

After 36-month, 34 subjects with a total of 178 study teeth (18 subjects, 89 teeth in control group; 16 subjects, 89 teeth in CPP-ACP group) remained. A total of seven participants dropped out between $\mathrm{T} 1$ and $\mathrm{T} 2$, four from the CPP-ACP group and three from the control group. The gender ratio, participant age, and duration of treatment with the MB appliance before investigation were not statistically significantly different between two groups $(p>0.05)$. The WSLs had a mean DD reading at baseline of $12.45 \pm 6.52$ in control group and $13.06 \pm 5.90$ in CPP-ACP group, which decreased at $8.20 \pm 4.38$ and $4.76 \pm 2.48$, respectively, at the 36 -month follow-up visit (Table 1 ). The mean baseline DD readings in the two groups were similar $(p>0.05)$. There was statistically significant differences between the mean DD readings of the two groups at the 36 -month $(p<0.001)$. The mean DMFT-DMFS increased were $1.8-2.6$ in the CPP-ACP group and 1.6-2.7 in control group $(p>0.05)$. Results of two groups, significantly decrease in salivary flow rates were obtained in each group $(p<0.05$, Table 1$)$.

According to Gorelick index, the severity of the lesions decreased significantly by $67.4 \%$ in test group and $57.3 \%$ in control group. The percentages of lesions that had regressed at T2 for both groups are shown Table 2. A statistically significant $(p<0.05)$ regression of the lesion activity was obtained in both study groups compared to T1, but there was no difference between the study groups $(p>0.05)$. For all lesions, $20 \%$ more had regressed with the CPP-ACP cream than with the control group at 36 months, but these was not significant $(p=0.05)$. For CPP-ACP group, while $S$. mutans salivary counts were significantly decreased $(p<0.05)$, significant differences in Lactobacillus counts were not observed $(p>0.05)$ after 36-months (Table 3). Saliva buffer capacity did not changed significantly after 36-month $(p>0.05)$. No side or adverse effects were reported in connection with the study. 
Table 1 Mean DD readings, salivary flow rate, DMFT and DMFT at two points

\begin{tabular}{|c|c|c|c|c|c|}
\hline & & Control & CPP-ACP & $\begin{array}{l}\text { Between } \mathrm{T} 1 \\
\text { and } \mathrm{T} 2\end{array}$ & $\begin{array}{c}\text { Group measurement } \\
\text { interaction significance value }\end{array}$ \\
\hline \multirow{2}{*}{$\mathrm{DD}$} & $\mathrm{T} 1$ & $12.45 \pm 6.52^{\mathrm{a}}$ & $13.06 \pm 5.90^{\mathrm{a}}$ & & \\
\hline & $\mathrm{T} 2$ & $8.20 \pm 4.38^{\mathrm{b}}$ & $4.76 \pm 2.48^{\mathrm{c}}$ & $<0.001$ & $<0.001$ \\
\hline Between groups & \multicolumn{4}{|c|}{0.006} & \\
\hline \multirow{2}{*}{ Salivary Flow Rate } & $\mathrm{T} 1$ & 1.9 & 1.8 & & \\
\hline & $\mathrm{T} 2$ & 1.3 & 1.4 & $<0.001$ & 0.161 \\
\hline Between groups & \multicolumn{3}{|c|}{0.750} & & \\
\hline \multirow{2}{*}{ DMFS } & $\mathrm{T} 1$ & $7.5 \pm 3.8$ & $8.0 \pm 4.2$ & \multirow{3}{*}{$<0.001$} & \multirow{3}{*}{0.976} \\
\hline & $\mathrm{T} 2$ & $10.2 \pm 4.8$ & $10.6 \pm 5.8$ & & \\
\hline Between groups & & & & & \\
\hline \multirow{2}{*}{ DMFT } & $\mathrm{T} 1$ & $5.8 \pm 2.5$ & $6.3 \pm 2.9$ & \multirow{3}{*}{$<0.001$} & \multirow{3}{*}{0.743} \\
\hline & $\mathrm{T} 2$ & $7.4 \pm 3.4$ & $8.1 \pm 4.0$ & & \\
\hline Between groups & \multicolumn{3}{|c|}{0.593} & & \\
\hline
\end{tabular}

Means with the same superscript letter are not statistically different from each other $(p>0.05)$.

Table 2 The changing of lesions according to baseline

\begin{tabular}{|c|c|c|c|c|c|c|c|}
\hline & WSL & Improvement & Control & CPP-ACP & $\mathrm{OR}^{\mathrm{a}}$ & $95 \% \mathrm{CI}$ & $p$ \\
\hline \multirow{9}{*}{ Severity } & \multirow{3}{*}{ All } & Worsened & $6(6.7)$ & $3(3.3)$ & \multirow{3}{*}{1.54} & \multirow{3}{*}{$(0.86,2.74)$} & \multirow{3}{*}{$>0.05$} \\
\hline & & Unchanged & $32(36.0)$ & $26(29.3)$ & & & \\
\hline & & Improved & $51(57.3)$ & $60(67.4)$ & & & \\
\hline & \multirow{3}{*}{ Slight } & Worsened & $0(0.0)$ & $0(0.0)$ & \multirow{3}{*}{2.01} & \multirow{3}{*}{$(1.06,3.82)$} & \multirow{3}{*}{$>0.05$} \\
\hline & & Unchanged & $18(34.1)$ & $10(20.4)$ & & & \\
\hline & & Improved & 35 (65.9) & $39(79.6)$ & & & \\
\hline & \multirow{3}{*}{ Severe } & Worsened & $6(16.6)$ & $3(7.5)$ & \multirow{3}{*}{1.37} & \multirow{3}{*}{$(0.78,2.39)$} & \multirow{3}{*}{$>0.05$} \\
\hline & & Unchanged & $14(38.8)$ & $16(40.0)$ & & & \\
\hline & & Improved & $16(44.6)$ & $21(52.5)$ & & & \\
\hline \multirow{9}{*}{ Activity } & \multirow{3}{*}{ All } & Worsened & $4(4.4)$ & $2(2.2)$ & \multirow{3}{*}{2.07} & \multirow{3}{*}{$(1.07,4.02)$} & \multirow{3}{*}{$>0.05$} \\
\hline & & Unchanged & $24(26.9)$ & $14(15.8)$ & & & \\
\hline & & Improved & $61(68.7)$ & $73(82.0)$ & & & \\
\hline & \multirow{3}{*}{ Inactive } & Worsened & $0(0.0)$ & $0(0.0)$ & \multirow{3}{*}{1.52} & \multirow{3}{*}{$(0.83,2.77)$} & \multirow{3}{*}{$>0.05$} \\
\hline & & Unchanged & $4(36.0)$ & $3(27.0)$ & & & \\
\hline & & Improved & $7(64.0)$ & $8(73.0)$ & & & \\
\hline & \multirow{3}{*}{ Active } & Worsened & $4(5.3)$ & $2(2.6)$ & \multirow{3}{*}{2.22} & \multirow{3}{*}{$(1.12,4.36)$} & \multirow{3}{*}{$=0.05$} \\
\hline & & Unchanged & $20(25.7)$ & $11(14.1)$ & & & \\
\hline & & Improved & $54(69.0)$ & $65(83.3)$ & & & \\
\hline
\end{tabular}

a The odds of a WSL regressing with CPP-ACP compared with the odds of a WSL regressing with fluoridated toothpaste. A statistically significant $(p<0.05)$ regression of the lesion activity and severity was obtained in both study groups compared to $\mathrm{T} 1$, but there was no difference between the study groups $(p>0.05)$. 
Table 3 Salivary S. mutans, Salivary Lactobacillus, Salivary buffer capacity (subject number) at two points

\begin{tabular}{|c|c|c|c|c|c|c|c|c|}
\hline & \multicolumn{3}{|c|}{ Control } & \multicolumn{3}{|c|}{ CPP-ACP } & \multicolumn{2}{|c|}{ Between Groups } \\
\hline & $\mathrm{T} 1$ & $\mathrm{~T} 2$ & $p$ & $\mathrm{~T} 1$ & $\mathrm{~T} 2$ & $p$ & $\mathrm{~T} 1$ & $\mathrm{~T} 2$ \\
\hline \multicolumn{9}{|l|}{ Salivary S. mutans } \\
\hline $0-10^{3} \mathrm{CFU}$ & 0 & 0 & \multirow{4}{*}{0.790} & 0 & 4 & \multirow{4}{*}{$<0.001$} & \multirow{4}{*}{0.446} & \multirow{4}{*}{0.040} \\
\hline $10^{3}-10^{4} \mathrm{CFU}$ & 5 & 6 & & 2 & 5 & & & \\
\hline $10^{4}-10^{5} \mathrm{CFU}$ & 6 & 6 & & 7 & 5 & & & \\
\hline $10^{5} \mathrm{CFU}$ & 7 & 6 & & 7 & 2 & & & \\
\hline \multicolumn{9}{|c|}{ Salivary Lactobacillus } \\
\hline $0-10^{3} \mathrm{CFU}$ & 0 & 0 & \multirow{4}{*}{0.753} & 0 & 0 & \multirow{4}{*}{0.317} & \multirow{4}{*}{0.694} & \multirow{4}{*}{0.808} \\
\hline $10^{3}-10^{4} \mathrm{CFU}$ & 3 & 5 & & 5 & 5 & & & \\
\hline $10^{4}-10^{5} \mathrm{CFU}$ & 9 & 7 & & 5 & 4 & & & \\
\hline $10^{5} \mathrm{CFU}$ & 6 & 6 & & 6 & 7 & & & \\
\hline \multicolumn{9}{|l|}{ Buffer Capacity } \\
\hline High & 6 & 4 & \multirow{3}{*}{0.274} & 6 & 5 & \multirow{3}{*}{0.999} & \multirow{3}{*}{0.858} & \multirow{3}{*}{0.390} \\
\hline Medium & 8 & 7 & & 5 & 7 & & & \\
\hline Low & 4 & 7 & & 5 & 4 & & & \\
\hline
\end{tabular}

\section{DISCUSSION}

In this study, WSLs that formed around fixed appliances during orthodontic treatment were followed longitudinally for 36 months after de-bonding. During this period, both the WSL severity and activity decreased in test and control groups, but only DD readings decreased in CPP-ACP group, indicating remineralization of the subsurface caries lesion. For ethical reasons, all participants were given fluoride toothpaste for use twice daily. All participants received oral hygiene monitoring and prophylaxes each 6 monthly appointments.

The tooth surfaces with WSLs of the subjects were assessed by a DD, and the readings which estimated the organic content and bacterial metabolites in enamel caries lesions were used to indicate the changes of the WSLs after CPP-ACP application. One study reported that DIAGNOdent had acceptable sensitivity (75\%) and high specificity (96\%) for their enamel depth analysis in smooth surface caries, as compared with quantitative light-induced fluorescence $(\mathrm{QLF})^{20)}$. Similarly, high sensitivity and acceptable specificity were also observed in other studies ${ }^{21,22)}$. The greater decrease in DD readings for lesions in test group compared to control may be taken to indicate a larger reversal of the early caries process due to the repeated CPP-ACP application. Several methods had been used for screening WSLs after orthodontic treatment such as QLF, DIAGNOdent or clinical scores ${ }^{11-16)}$. On the other hand the DIAGNOdent readings should be interpreted with caution because statistically significant differences might not necessarily have clinical significance.

The evaluation of biological and cosmetic changing of WSLs aimed in this project, so laser fluorescence and clinical scores were used together. ICDAS II authorizes for the estimation of caries lesion at the initial level attributed to visual evaluation of the tooth surface and has been indicated to have content and correlational currency with histological lesion depth ${ }^{18)}$. We used ICDAS II for activity evaluation of free smooth surfaces in this study. Clinical investigation were taken into consideration for detecting enamel lesion activity were based on a modification of the Bailey et al. caries lesion activity assessment criteria and implicate visual appearance, tactile sensation and potential for plaque accumulation $^{15)}$. The Gorelick Index to screen WSL is so far the most utilized procedure to record WSL in orthodontic patients. This index is great appropriate to enrolled the certain area of WSL but may have restrictions in evaluating the content of WSL.

Both in-vitro and in-situ studies have demonstrated that CPP-ACP can promote the remineralization of subsurface enamel lesions ${ }^{10,12)}$ Thirty six weeks after debonding in our study, the lesions showed significant improvement with respect to fluorescence loss, as monitored by DD. However, both test and control methods resulted in significant improvements of WSLs severity and activity scores compared to baseline, but no significant differences were detected between the groups after three years. Some studies found that CPP-ACP is useful agent for regression of WSL ${ }^{13,15)}$, but others not $^{14,16)}$. In our study, it can be suggested that CPPACP was effective for remineralization of subsurface lesion after usage 36 months, but it was not difference to improvement of surface severity and activity from normal fluoridated toothpaste. The appearance of the WSLs did not improve well in CPP-ACP group than the 
placebo control group. The variation of different results about CPP-ACP among studies could be attributed to differences in the number of teeth examined (or subjects), the methods and the standardizations of examinations, the location of the study sample (cultural or socioeconomic differences), time era of the study, treatment or follow duration, and materials.

According to the our results, it can be concluded that long term usage of CPP-ACP with 1,450 ppm fluoridated toothpaste had synergistic impact on subsurface lesion with regression. Beerens et al. reported that CPP-ACP with fluoride (CPP-ACPF) has the same potential as CPP-ACP but the additional benefits of supplemental fluoride $^{16)}$. Using CPP-ACP with fluoridated toothpaste could be remineralize subsurface lesions by forming fluorapatite within the lesion. Our results have led to the application of CPP-ACP with fluoridated toothpaste, for use supplementary to the normal daily oral hygiene procedure. On the other hand $1,450 \mathrm{ppm}$ fluoridated toothpaste was found to be effective for decreasing only lesion severity and activity after 36 months.

It is well known that past caries experience is the strongest predictor of future caries development among school children and adolescents ${ }^{23}$. Since the incidence and prevalence of caries is greater in fixed orthodontic patients, clinicians need a head start in this field such as various tools and methods other than clinical findings to predict the occurrence of new caries before frank cavitation ${ }^{24)}$. DMFT and DMFS used in this study for monitoring subject's caries risk, and we found that each parameter increased significantly in two groups. Different factors can modify subject's caries risk such as past caries experience, oral health habits, saliva secretion rate, diet frequency of type, socioeconomic levels ${ }^{25)}$.

Clinically significant changes in salivary functions may be considered an etiologic factor that contributes to the development or prevention of dental caries. According to our results significantly decreased in salivary flow rates were obtained after 36 weeks, but buffer capacity not changed significantly. It is well known that orthodontic appliances increase the stimulated salivary flow rate ${ }^{26)}$. One study reported that salivary flow rate is more sensitive to the placement of orthodontic appliances than buffer capacity ${ }^{27)}$. Such a modification can be considered as a physiological response to the mechanical stimulation resulting from the presence of the fixed orthodontic appliances, as a result of a disturbed intraoral homeostasis ${ }^{28)}$. However, it was reported that the beneficial effects of CPP-ACP may be attributed to an increased salivary buffering effect, but it is not supported in our findings.

We used saliva and dip-slide test for evaluate $S$. mutans and Lactobacillus ${ }^{29)}$. Beerens et al. reported that the percentages of aciduric bacteria and of S. mutans decreased significantly both CPP-ACFP and control groups, but they were found no differences between groups 12 weeks after de-bonding. Our bacterial results should be interpreted with caution because of dropout rate. The study period of 36 weeks might be regarded as long compared with other studies ${ }^{11-16)}$, so the dropout rate in this study is relatively high (17\%). So we failed to detect any positive or negative effects of CPP-ACP on saliva bacterial level $\mathrm{s}^{30}$.

\section{CONCLUSIONS}

Considering the limitations of any in vivo study, the following clinical conclusions can be drawn.

1. The laser fluorescence level of WSLs can reduce over time; CPP-ACP support increased remineralization of the demineralized enamel surface. On the other hand only 1,450 ppm fluoridated toothpaste was found to be effective for decreasing lesion severity and activity after 36 months.

2. CPP-ACP did not appear to be more effective than only $1,450 \mathrm{ppm}$ fluoridated toothpaste for improving the appearance of WSLs after 36 months.

3. These type lesions are not easily remineralized may require a different approach or new material to perform both biologic and aesthetic rehabilitation.

\section{REFERENCES}

1) Oral microbiological changes, long-term enamel alterations due to decalcification, and caries prophylactic aspects. In: Brantley WA, Eliades T, editors. Orthodontic materials. Scientific and clinical aspects. Stuttgart (Germany): Thieme; 2001.

2) Chang HS, Walsh LJ, Freer TJ. Enamel demineralization during orthodontic treatment. Aetiology and treatment. Aust Dent J 1997; 42: 322-327.

3) Øgaard B, Rølla G, Arends J. Orthodontic appliances and enamel demineralization. Part 1. Lesion development. Am J Orthod 1988; 94: 68-73.

4) Øgaard B. Prevalence of white spot lesions in 19-year-olds: a study on untreated and orthodontically treated persons 5 years after treatment. Am J Orthod Dentofacial Orthop 1989; 96: 423-427.

5) Van der Veen MH, Mattousch T, Boersma JG. Longitudinal development of caries lesions after orthodontic treatment evaluated by quantitative lightinduced fluorescence. Am J Orthod Dentofacial Orthop 2007; 131: 223-228.

6) Paschos E, Kleinschrodt T, Clementino-Luedemann T, Huth KC, Hickel R, Kunzelmann KH, Rudzki-Janson I. Effect of different bonding agents on prevention of enamel demineralization around orthodontic brackets. Am J Orthod Dentofacial Orthop 2009; 135: 603-612.

7) Stecksen-Blicks C, Renfors G, Oscarson ND, Bergstrand F, Twetman S. Caries-preventive effectiveness of a flüoride varnish: a randomized controlled trial in adolescents with fixed orthodontic appliances. Caries Res 2007; 41: 455-459.

8) Lundstrom F, Krasse B. Streptococcus mutans and lactobacilli frequency in orthodontic patients; the effect of chlorhexidine treatments. Eur J Orthod 1987; 9: 109-116.

9) Reynolds EC. Remineralization of enamel subsurface lesions by casein phosphopeptide-stabilized calcium phosphate solutions. J Dent Res 1997; 76: 1587-1595.

10) Walker G, Cai F, Shen P, Reynolds C, Ward B, Fone C, Honda S, Koganei M, Oda M, Reynolds E. Increased remineralization of tooth enamel by milk containing added casein phosphopeptide-amorphous calcium phosphate. J 
Dairy Res 2006; 73: 74-78.

11) Huang GJ, Roloff-Chiang B, Mills BE, Shalchi S, Spiekerman C, Korpak AM, Starrett JL, Greenlee GM, Drangsholt RJ, Matunas JC. Effectiveness of MI Paste Plus and PreviDent fluoride varnish for treatment of white spot lesions: a randomized controlled trial. Am J Orthod Dentofacial Orthop 2013; 143: 31-41.

12) Sithisettapong T, Doi T, Nishida Y, Kambara M, Phantumvanit P. Effect of CPP-ACP paste on enamel carious lesion of primary upper anterior teeth assessed by quantitative light-induced fluorescence: A one-year clinical trial. Caries Res 2015; 49: 434-441.

13) Andersson A, Skold-Larsson K, Hallgren A, Petersson LG, Twetman S. Effect of a dental cream containing amorphous calcium phosphate complexes on white spot lesion regression assessed by laser fluorescence. Oral Health Prev Dent 2007; 5: 229-233.

14) Brochner A, Christensen C, Kristensen B, Tranaeus S, Karlsson L, Sonnesen L, Twetman S. Treatment of postorthodontic white spot lesions with casein phosphopeptidestabilised amorphous calcium phosphate. Clin Oral Investig 2011; 15: 369-373.

15) Bailey DL, Adams GG, Tsao CE, Hyslop A, Escobar K, Manton DJ, Reynolds EC, Morgan MV. Regression of post-orthodontic lesions by a remineralizing cream. J Dent Res 2009; 88: 11481153.

16) Beerens MW, van der Veen MH, van Beek H, ten Cate JM. Effects of casein phosphopeptide amorphous calcium fluoride phosphate paste on white spot lesions and dental plaque after orthodontic treatment: a 3-month follow-up. Eur J Oral Sci 2010; 118: 610-617.

17) Gorelick L, Geiger AM, Gwinnet AJ. Incidence of white spot formation after bonding and banding. Am J Orthod 1982; 81: 93-98.

18) Almosa NA, Lundgren T, Bresin A, Birkhed D, Kjellberg H. Diagnosing the severity of buccal caries lesions in orthodontic patients at de-bonding using digital photographs. Acta Odontol Scand 2014; 72: 474-480.

19) World Health Organization. Oral health surveys, basic methods. 4th ed. Geneva: 1997.

20) Shi XQ, Tranaeus S, Angmar-Månsson B. Comparison of QLF and DIAGNOdent for quantification of smooth surface caries. Caries Res 2001; 35: 21-26.

21) Rocha RO, Ardengi TM, Oliveria LB, Rodrigues CR, Ciamponi AL. In vivo effectiveness of laser fluorescence compared to visual inspection and radiography for the detection of occlusal caries in primary teeth. Caries Res 2003; 37: 437-441.

22) Anttonen V, Seppa L, Hausen H. Clinical study of the use of the laser fluorescence device DIAGNOdent for detection of occlusal caries in children. Caries Res 2003; 37: 17-23.

23) Pitts NB. Do we understand which children need and get appropriate dental care? Br Dent J 1997; 182: 273-278.

24) Karadaş M, Cantekin K, Celikoglu M. Effects of orthodontic treatment with a fixed appliance on the caries experience of patients with high and low risk of caries. J Dent Sci 2011; 6: 195-199.

25) Reich E, Lussi A, Newbrun E. Caries risk assessment. Int Dent J 1999; 49: 15-26.

26) Chang HS, Walsh LJ, Freer TJ. The effect of orthodontic treatment on salivary flow, $\mathrm{pH}$, buffer capacity, and levels of mutans streptococci and lactobacilli. Aust Orthod J 1999; 15: 229-234.

27) Lara-Carrillo E, Montiel-Bastida NM, Sánchez-Pérez L, Alanís-Tavira J. Effect of orthodontic treatment on saliva, plaque and the levels of Streptococcus mutans and Lactobacillus. Med Oral Patol Oral Cir Bucal 2010; 15: 924929.

28) Alessandri Bonetti G, Incerti Parenti S, Garulli G, Gatto MR, Checchi L. Effect of fixed orthodontic appliances on salivary properties. Prog Orthod 2013; 14: 13.

29) Pinelli C, Serra MC, Loffredo LC. Efficacy of a dip slide test for mutans streptococci in caries risk assessment. Community Dent Oral Epidemiol 2001; 29: 443-448.

30) Chen H, Liu X, Dai J, Jiang Z, Guo T, Ding Y. Effect of remineralizing agents on white spot lesions after orthodontic treatment: a systematic review. Am J Orthod Dentofacial Orthop 2013; 143: 376-382. 